\title{
TECNO REDENCIÓN DE CUERPOS TRANSEXUALES: APROPIACIÓN TECNOLÓGICA Y AUTOGESTIÓN DE IDENTIDADES INCONCLUSAS
}

\author{
Alejandra Roca ${ }^{1}$ \\ María Alejandra Dellacasa ${ }^{2}$
}

\begin{abstract}
RESUMEN
En este trabajo nos proponemos indagar acerca de las intervenciones y procedimientos que arbitran límites entre las nociones de cuerpo, sexualidad y naturaleza. Basado en un trabajo etnográfico en instituciones públicas de Buenos Aires y La Plata (Argentina), se plantean cuestiones respecto de los procesos médicolegales de "reasignación de sexo" en personas transexuales, que iluminan la forma en que se despliegan sentidos sobre la plasticidad y precariedad de las "esencias humanas", en tanto tensión entre lo que las personas son (identidad) y lo que quieren llegar a ser (proyecto). Se debaten las propuestas del transhumanismo, entendiendo que la dimensión soteriológica de las Biociencias habilita las esperanzas de tecno-redención. Por último se pretende aportar una reflexión sobre el rol de las categorías científicas y el contenido político de la emergencia de identidades y resistencias colectivas a la tutela del control cognitivo y material de las Biociencias.
\end{abstract}

Palabras clave: Biociencias. Transexualidad. Cuerpo. Identidades cyborgs.

\footnotetext{
${ }^{1}$ Docente e investigadora del Instituto de Ciencias Antropológicas, Universidad de Buenos Aires, Argentina.roca.ale@gmail.com

${ }^{2}$ Candidata a Doctora por el Instituto de Ciencias Antropológicas, Universidad de Buenos Aires. Docente de la Escuela Superior de Ciencias de la Salud, Universidad Nacional del Centro de la Provincia de Buenos Aires, Argentina. maledellacasa@yahoo.com.ar
} 


\title{
TECNORREDENÇÃO DE CORPOS TRANSEXUAIS. APROPRIAÇÃO TECNOLÓGICA E AUTOGESTÃO DE IDENTIDADES INCONCLUSAS \\ RESUMO
}

Neste trabalho nos propomos indagar as intervenções e procedimentos que arbitram os limites entre noções de corpo, sexualidade e natureza. Baseado em um trabalho etnográfico em instituições públicas de Buenos Aires e La Plata (Argentina), formulam-se questões a respeito dos processos médico-legais de "redesignação sexual" em pessoas transexuais, que ilustram a forma em que se desdobram sentidos sobre a plasticidade e precariedade das "essências humanas" enquanto tensão entre 0 que as pessoas são (identidade) e o que querem ser (projeto). São discutidas as propostas do transhumanismo, entendendo que a dimensão soteriológica das biociências habilita as esperanças da tecnorredenção. Em última instância, pretendese contribuir com a reflexão acerca do papel das categorias científicas e do conteúdo político da emergência de identidades e resistências coletivas sobre a tutela do controle cognitivo e material das biociências.

Palavras-chave: Biociências. Transexualidade. Corpo. Identidades ciborgues.

\section{TECHNO-REDEMPTION OF TRANSSEXUAL BODIES: APPROPRIATION OF TECHNOLOGY AND SELF-MANAGEMENT OF UNFINISHED IDENTITIES}

\begin{abstract}
In this paper, we want to explore the modifications and procedures that delineate limits between the notions of body, sexuality, and nature. Based on ethnographic fieldwork in public institutions of Buenos Aires and La Plata (Argentina), we examine the medical-legal process concerning "sex reassignment" in transsexual people, which illuminates the unfold senses about the plasticity and precariousness of 'human essences', as well as the tensions between what human is (identity) and what he/she wants to be (project). We discuss the proposals of transhumanism, accepting that the soteriological dimension of biosciences enable the hopes of techno-redemption. Finally, we intend to contribute with a reflection about the role of scientific categories and the political perspective of the emergence of identities and collective resistances about the tutelage of cognitive and material control of the biosciences.
\end{abstract}

Keywords: Bioscience. Transsexuality. Body. Cyborg identities 
[el hombre] tiene el poder de crear otras naturalezas, otros cursos, otros órdenes a través de su inteligencia [...] el hombre al final podría hacerse a sí mismo dios en la tierra. (Giordano Bruno, 1584) ${ }^{3}$

\section{INTRODUCCIÓN}

En esta contribución intentamos sintetizar parte de las discusiones que hemos ido desarrollando al interior del equipo de investigación de Antropología y Biociencias, un proyecto de la Universidad de Buenos Aires ${ }^{4}$, que nuclea investigadores y tesistas en líneas de trabajo diferentes, pero complementarias entre sí. Los ejes de indagación de este espacio articulan aportes y reflexiones vinculadas con las transformaciones y torsiones que habilita la introducción de tecnologías en la salud y el cuerpo, particularmente aquellas intervenciones y procedimientos que arbitran límites entre las nociones de cuerpo, sexualidad y naturaleza.

La relación biología/sociedad, naturaleza/cultura constituye uno de los principales nudos problemáticos de la teoría antropológica, que trascendió las fronteras disciplinares otorgándole protagonismo en los debates sobre raza, sexualidad y construcción biomédica del cuerpo. En las últimas décadas los dogmas anclados en los dualismos ontológicos modernos (cuerpo/mente, femenino/masculino, sujeto/objeto, vivo/muerto, entre otros) fueron interpelados a partir de una renovación teórica surgida de enfoques y articulaciones provenientes de diversos campos empíricos. Desde la antropología médica, la historia de la antropología y la antropología de la ciencia y la tecnología, los esfuerzos por abordar la complejidad de estas problemáticas - que focalizan el espesor material de los artefactos - reclaman de una articulación disciplinar que ha ido prosperando en los últimos años.

En tanto los cuerpos son el epicentro material de estas maniobras de intervención tecno-científica, la mirada retrospectiva de los sentidos con que se ha ido construyendo el cuerpo en la biomedicina y en la ciencia en general, nos revela una trama de potentes significados. Desde la reducción de cuerpos a espécimen para la exhibición y el aprendizaje en los museos y/o las cátedras de

\footnotetext{
${ }^{3}$ En Diaz Cruz (2009, p.34)

${ }^{4}$ El Proyecto "Cuerpos, raza y sexualidades: Procesos de producción y legitimación del conocimiento en Antropología y Biociencias. Una aproximación genealógica a la construcción de categorías, dispositivos y artefactos" se desarrolla en el seno del Programa de Antropología y Salud de la Facultad de Filosofía y Letras de la Universidad de Buenos Aires.
} 
anatomía, hasta los protocolos de hormonización (HRT - Hormone Replacement Therapy) y las cirugías de transformación corporal, la ciencia ha tenido un lugar preponderante en la denominación, la jerarquización semántica y performativa y, en general, en el diseño de nuevos horizontes en las expectativas de la sociedad contemporánea.

Ya sea desde los profesionales/practicantes o desde la perspectiva de los usuarios/demandantes, las prácticas se orientan en un sentido unívoco. Todas convergen en el fundamentalismo tecnológico que favorece una suerte de tecnoredención o soteriología de las biociencias que abriga las mayores y más insólitas esperanzas hacia el futuro inmediato. En este trabajo se discuten estas perspectivas a la luz del trabajo de campo realizado entre los años 2009 y 2012, en las ciudades de Buenos Aires y La Plata, en Argentina, en el contexto de una etnografía entre profesionales de la salud en instituciones públicas, vinculados con los procesos de "reasignación de sexo" en personas transexuales (DELLACASA, 2013a).

Trabajamos con entrevistas reiteradas en profundidad a los distintos profesionales que integran los equipos de salud y recurrimos a instancias de observación en los espacios de atención, como salas de espera, consultorios y office de cirugía donde tienen lugar ateneos y suelen desarrollarse distintos intercambios entre integrantes de los equipos acerca de los "casos". El trabajo de campo de más de tres años desarrollado en dos hospitales (los primeros en el país), nos permitió reconstruir experiencias profesionales y personales a la vez que indagar en torno a la construcción de categorías cognitivas, rutinas y procedimientos de intervención. Nos propusimos reconstruir el dispositivo médico-legal de la transexualidad, tomando como punto de partida los procesos y las relaciones entre usuarios e instituciones de salud.

\section{LA MIRADA MODERNA DEL CUERPO}

Es preciso revisar el devenir de la construcción del cuerpo como objeto de la biomedicina para situar y comprender de manera más precisa la forma en que la modernidad arrasa las versiones sacras, integradas y monistas del cuerpo. El cuerpo como "objeto de estudio", encarnado en el cadáver de la disección, ha sido sistemáticamente desmantelado de sus dimensiones históricas y contextuales; se convierte en un objeto natural - perteneciente al mundo de las cosas - es decir, pasible de ser investigado, manipulado, intervenido. En cierta forma, el cuerpo expresa todas las dimensiones de la investigación de la ciencia moderna, 
representa una indagación sistemática, replicable y pública, ${ }^{5}$ subsumido en la lógica mecánica que prima en la explicación de los fenómenos de la naturaleza. Mediante un movimiento de secularización, la lógica occidental ha ido asociando paulatinamente el cuerpo al "poseer" y no al "ser", convirtiéndolo en otro de los "hechos" de la naturaleza.

En síntesis, la concepción moderna del cuerpo se presenta asociada al ascenso del individualismo como estructura social y a la emergencia del pensamiento dualista, mecanicista y laico sobre la naturaleza; cristalizando la construcción biomédica como protagonista en su trayectoria. En tanto las prácticas de la medicina conservan "el secreto del cuerpo" a través de la lectura de signos a partir de los sentidos - tocar, palpar, oler, escuchar, la tecnología ha permitido volver visible lo invisible, exhibiendo una nueva cartografía corporal interna, infinitamente fragmentada y, por qué no, fascinante (ROCA, 2010).

En este esquema, el cuerpo como unidad integral y como objeto de estudio fue desapareciendo, disolviéndose en unidades de intervención cada vez más fragmentadas, incluso separadas del resto del cuerpo. La metáfora troncal del cuerpo como máquina, junto a una concepción de la naturaleza como materia prima maleable, productora de riqueza y perfectible, autorizaron una serie de prácticas vinculadas con las biotecnologías, en las que subyace la noción del cuerpo como "suma orgánica" de fragmentos ensamblados. De allí que Haraway (1991) entienda que la biología contemporánea ha sufrido una trasformación de "ciencia de organismos sexuales a ciencia de ensamblajes genéticos".

A partir de la Segunda Guerra Mundial pueden rastrearse una serie de eventos que produjeron modificaciones en los mecanismos de control y gestión del cuerpo, alterando el paradigma que propuso inicialmente Foucault (1995): ya no se trata de la sociedad disciplinaria en la que la arquitectura y la ortopedia servían para entender la relación cuerpo-poder. Beatriz Preciado (2008) sostiene que del viejo modelo "panóptico" de control arquitectónico que determina los cuerpos desde afuera, se ha pasado a un nuevo modelo sintético que trabaja desde el interior del cuerpo, casi invisible, modificando la misma composición química del individuo.

\footnotetext{
${ }^{5}$ Hasta el siglo XV la Iglesia Católica prohibía explícitamente las disecciones y cualquier otra manipulación de cadáveres con fines profanos, por el contrario, los cuerpos y fragmentos corporales de santos y mártires eran conservados, exhibidos y venerados. En tanto en el siglo XVI se popularizan los primeros teatros anatómicos, convirtiéndose en espectáculo con el más variado auditorio.
} 
El modelo de acción sobre el cuerpo es la microprostética: el poder actúa a través de una molécula que viene a formar parte de nuestro sistema inmunitario, de la silicona que toma la forma de senos, de un neurotransmisor que modifica nuestra forma de percibir y actuar, de una hormona y su acción sistémica sobre el hambre, el sueño, la excitación sexual, la agresividad o la descodificación social de nuestra feminidad y masculinidad. (PRECIADO, 2008, p. 67).

\section{BIOMEDICINA Y EXPERIENCIA TRANSEXUAL}

Desde mediados del siglo $\mathrm{XX}$, ha venido conformándose un campo específico de saberes y prácticas que habilitan diferentes intervenciones biomédicas, dando lugar a la "reescritura" o "corrección" de la naturaleza del cuerpo. Estas prácticas estandarizadas reformularon las categorías de cuerpo, sexo, género e identidad, regulando los tránsitos y relaciones que requieren a su vez de redefiniciones legales para el ejercicio de los derechos civiles. La paulatina desnaturalización y la creciente producción de conocimiento médico científico respecto de qué es la transexualidad y qué es "ser transexual" habilitó la creación de significación, allí donde sólo había un puñado de experiencias. Poco a poco la individuación moderna del "síndrome" (FRIEDSON, 1978) permitió ir delimitando un conjunto de signos leídos como "síntomas", diferenciándolo, a la vez, de otras sexualidades no heterosexuales.

Para "producir" una mirada moderna de la transexualidad, en términos biomédicos, era necesario partir de una determinada concepción de cuerpo, de "cuerpo normal" en relación tanto a sus órganos, como a sus prácticas. A la vez, se ha hecho necesario también, una determinada representación de "cuerpo transexual" pasible de ser intervenido ${ }^{6}$. Las intervenciones corporales pueden contribuir y/o ser utilizadas para "desestabilizar" las nociones de sujeto fijo e inmutable; coincidiendo con la celebración de la disrupción de los contornos estables del cuerpo, como una precondición importante para cuestionar y desestabilizar las construcciones de género represivas (BUTLER, 2001).

Las cirugías y otras intervenciones corporales forman parte de las estrategias de las personas transexuales para lograr sentirse "cómodas" en sus cuerpos, "un sujeto con un cuerpo, en lugar de sólo 'cuerpos'; paradójicamente, las técnicas quirúrgicas permiten a estas personas devenir en sujetos corporizados, en lugar de cuerpos objetivados" (DAVIS, 1995 p. 161).

\footnotetext{
${ }^{6}$ De acuerdo a los trabajos de Harry Benjamin (1966) la opción "terapéutica" que ofrece la biomedicina es la de adaptar el cuerpo, ante la imposibilidad de adaptar la mente.
} 


\section{CUERPOS INTERVENIDOS Y TRANSHUMANISMO}

Las intervenciones vinculadas con la transexualidad despliegan debates que se centran en la plasticidad y precariedad de la llamada "esencia humana" en tanto tópico propio de la filosofía de la tecnología y de la filosofía de la ciencia, como tensión entre lo que el hombre es (identidad) y lo que quiere llegar a ser (proyecto). Nick Bostrom recuerda la distinción Husserliana entre Körper, como el mero cuerpo físico, del Liebe, en tanto cuerpo apropiado, asimilado y experimentado como irrepetible, con su carga de voluntariedad y vitalidad. "Así, cuando la ciencia considera sólo el cuerpo físico (Körper) lo ve como dessubjetivizado y manipulable y no como vehículo de manifestación unitaria de la persona, abierto a la intencionalidad y la historicidad de sus acciones y a la identidad ontológica que le da base" (BOSTROM, 2011, p. 590). Este punto de partida abre las reflexiones del transhumanismo ${ }^{7}$ que supone una infraestructura biológica neutra y manipulable según criterios utilitaristas y funcionales; y una superestructura simbólica emocional.

En sus diferentes variantes la propuesta del transhumanismo supone la posibilidad de mejorar tecnológicamente a los seres humanos como individuos y como sociedad por medio de su manipulación como especie biológica; abrazando el sueño de abandonar y superar la precariedad de la existencia orgánica. Algunos autores, como Vilaça y Palma (2012), refieren la "odisea de la manipulación" como una búsqueda del "plus": más salud, más felicidad, más longevidad; 0 en Roca (2010) la observación de las publicidades y la información de los bancos de esperma y óvulos revela una "hipernormalidad" (más cantidad y movilidad de espermatozoides de lo normal, más controles que en la reproducción natural, etc.). En este sentido, en la criopersevación de gametos parece afirmarse una tendencia a "resguardar" ciertos atributos concebidos como valores estéticos 0 reaseguros de la salud, al estilo de una "caja de seguridad biológica" (ROCA, 2010).

El transhumanismo defiende un desarrollo responsable de las biotecnologías para ampliar y perfeccionar las capacidades físicas, mentales y emocionales; Max More (1990) lo definió como un proceso de perpetua

\footnotetext{
${ }^{7}$ La palabra "transhumanismo" fue usada por primera vez por Julian Huxley. En "Religion without revelation" (1927), escribió: "La especie humana puede, si lo desea, trascenderse a sí misma - no sólo esporádicamente, un individuo aquí de cierta manera, un individuo ahí de otra, sino en su totalidad, como humanidad. Necesitamos un nombre para esta nueva creencia. Tal vez transhumanismo servirá: el hombre permaneciendo hombre, pero transcendiéndose mediante la realización de nuevas posibilidades de y para su naturaleza humana".
} 
superación de los límites biológicos. El propio Bostrom ${ }^{8}$ presenta indicios sobre la continuidad entre la noción de sujeto del transhumanismo y el humanismo renacentista. En 1486 Giovanni Pico della Mirandola proclamó que el hombre no tiene una forma acabada y es responsable de dársela a sí mismo:

Te hemos hecho una criatura que no es ni del cielo ni de la tierra, ni mortal ni inmortal, para que puedas, como libre y orgulloso moldeador de tu propio ser, darte a tí mismo la forma que prefieras (Oración sobre la dignidad humana apud BOSTROM, 2011, p. 175).

Un transhumano es un "humano de transición", alguien que en virtud de su uso de la tecnología, valores culturales y modo de vida constituye un enlace evolutivo con la era de posthumanidad. Esfandiary describía en 1989 los signos del transhumano, que incluirían "prótesis, cirugía plástica, uso intensivo de telecomunicaciones, un perfil cosmopolita y un modo de vida trotamundos, andrógino, de reproducción mediada (tal como fertilización in vitro), ausencia de creencia religiosa, y un rechazo de los valores familiares tradicionales" (ESFANDIARY apud BOSTROM, 2011, p. 172). Los avances en psicofármacos y medicamentos para mejorar el tono muscular, borrar selectivamente recuerdos, activar la concentración, descansar o inundar con hormonas un cuerpo "equivocado" o que desea ser "otro", así como las intervenciones de la ingeniería y la consultoría genética, representan para los transhumanistas una opción para "rescatar al sapiens del ciego proceso de variación aleatoria, adaptación y selección al que lo habría arrojado la evolución, y dotarlo del poder de controlar las sucesivas fases de su desarrollo como especie" (BOSTROM, 2011, p. 191).

Manzocco (2014, p. 46) se refiere al transhumanismo como un extraño movimiento híbrido entre la ideología, la filosofía y la fe, que construye una nueva torre de Babel, en donde los humanos viviríamos como entidades electrónicas, a partir de algunos desarrollos que ya existen, tales como la realidad virtual, el diagnóstico genético pre-implantatorio, la ingeniería genética; los medicamentos

\footnotetext{
${ }^{8}$ Nick Bostrom y David Pearce crearon la Asociación Mundial Transhumanista (WTA), a principios de 1998, para proporcionar una base organizativa general para todos los grupos e intereses transhumanistas a lo largo del espectro político. Pearce (2004) defiende en The bedonistic imperative un ambicioso programa para eliminar el sufrimiento tanto en los animales humanos como en los no-humanos, por medio de la neuro-tecnología avanzada y propone un "paraíso ingenieril" en el que seres sentientes serían rediseñados para experimentar niveles de bienestar sin precedentes. En 2002, la WTA produjo The Transhumanist Declaration, disponible en: < http://transhumanism.org/index.php/WTA/declaration >
} 
que mejoran la memoria y la concentración, drogas para el rendimiento y los estados de ánimo; cirugías estéticas y de cambio de sexo, prótesis, hormonas, medicina anti-edad, interfaces humano-ordenador, entre otros, y a la espera de que "conforme maduren, la combinación de estas capacidades tecnológicas podría transformar profundamente la condición humana". La agenda transhumanista, será la de "hacer tales opciones de perfeccionamiento seguras y disponibles para cualquier persona" (BOSTROM, 2005, p. 14).

Ahora bien estos procesos de tecnologización también pueden ser entendidos como modos que asume la "política de gobierno de las poblaciones", en tanto el "diseño químico de los gustos y la bioregulación de los vínculos sociales implica la reaparición de viejas formas de esclavitud, servidumbre y sometimiento" (D'ODORICO, 2014, p. 8). El peligro y la angustia ambivalente de un mundo que funciona automáticamente y se reproduce cada vez con más autonomía, invade los espacios de reflexión y parece esfumar el contorno mismo de lo humano, como si fuéramos testigos de una etapa cuyas "experiencias medicalizadas, informatizadas y biológicamente intervenidas equiparan y superan los mundos literarios imaginados por William Bourroughs o James Ballard" (D'ODORICO, 2014, p. 8).

En un plano de flagrante oposición a las propuestas del transhumanismo, el bioconservadurismo, básicamente se opone al uso de la tecnología para modificar la naturaleza genética, biológica y estética de los seres humanos. El singular espectro que cubre el bioconservadurismo va desde religiosos de derecha, ecologistas y anti-globalizadores de izquierda. Como es habitual respecto de las concepciones de la tecnología en general (ROCA, 2006, p. 112 y 2007) las feministas se han situado en ambos lados del debate. Las ecofeministas han sospechado de la biotecnología para remodelar los cuerpos o controlar la reproducción, interpretando una extensión ideológica del patriarcado 0 , alternativamente, la han visto como un síntoma de una mentalidad obsesionada por el control, no empática, fascinada por los artefactos y negadora del cuerpo. En tanto, otras - como S. Firestone (1970) - han saludado el potencial liberador de la tecnología.

Entre los bioconservadores, la mayor preocupación es la accesibilidad, por ejemplo, a psicofármacos - antidepresivos, ansiolíticos, metanfetaminas, etc. y hormonas, responsables de provocar profundos cambios en lo más íntimo del ser humano, que pueden comprarse fácilmente en el mercado. Estas corrientes producen un cuestionamiento ético-moral, hacia el consumo cosmético 0 independiente de las indicaciones terapéuticas, bajo el nombre de medicalización 
de la normalidad. Es evidente que estas miradas, hacen caso omiso del consumo "político" y provocador de hormonas que documenta Preciado (2008), en tanto despliega una serie de experiencias que sirven de pretexto para la reflexión sobre las categorías y la tutela del control cognitivo - material retenido en las "indicaciones terapéuticas".

\section{TERAPIA HORMONAL “CRUZADA”. RESISTIR LA NORMALIZACIÓN EN LOS CUERPOS.}

El abordaje terapéutico de la experiencia transexual, propuesto por la biomedicina y expresado en los Standards of Care For Gender Identity Disorders (SOC's) ${ }^{9}$, contempla intervenciones corporales quirúrgicas (cirugía de reasignación de sexo, CRS) y hormonales (terapia hormonal de reemplazo, THR, 0 HTR según sus siglas en inglés). Nos centramos en estas últimas, con la intención de reflexionar respecto de las posibilidades de gestión biotecnológica que intervienen en la conformación del cuerpo y la sexualidad, a partir del análisis del dispositivo médico-legal de la transexualidad en el ámbito hospitalario en Argentina, que tomó como punto de partida los procesos y las relaciones entre usuarios e instituciones de salud, como fuera referido anteriormente.

La terapia hormonal encuentra su argumento en un modelo químico del sexo y del cuerpo. A principios del siglo XX la "esencia" de la feminidad fue localizada en una sustancia química: las hormonas sexuales. El fascinante trabajo de Nelly Oudshoorn (1994) desanda el recorrido que ha llevado a la biomedicina a concebir el concepto de hormonas sexuales, como una especie de "mensaje químico" de masculinidad y feminidad. En este sentido, la historia que ella narra acerca de la introducción de las hormonas en los discursos científicos, permite visibilizar el poder de transformación que opera la ciencia y la tecnología en la comprensión de nuestra cotidianeidad. La interpretación del cuerpo en términos de hormonas sexuales contribuye a una redefinición del mismo en términos de experiencias corporales, a la vez que habilita a ejercer un "control" sobre ciertos caracteres físicos. En "la química del cuerpo" los fármacos se cubren de cualidades "femeninas" o "masculinas", como si éstas fuesen capaces de producir los estereotipos culturales que caracterizan a ciertas identidades. "Muchas de las

\footnotetext{
${ }^{9}$ The World Professional Association for Transgender Health, anteriormente denominada Harry Benjamin International Gender Dysphoria Association (HBIGDA), elaboró desde 1969 las sucesivas versiones de los Standards of Care For Gender Identity Disorders (SOC's); la última es la séptima versión y data de 2011.
} 
diferencias en el comportamiento, funciones, papeles y características consideradas como típicamente masculinas o femeninas han sido atribuidas a las hormonas" (OUDSHOORN, 1994, p. 88).

Aún es incipiente la investigación y los protocolos médicos desarrollados acerca del tratamiento de reemplazo hormonal "cruzado"; suele proveerse a las transexuales femeninas una terapia estrogénica y antiandrogénica, en algunos casos también se les administra progesterona. Las hormonas más utilizadas en la THR, también llamada Terapia Hormonal Sustitutiva son: etil estradiol, estrágenos conjugados, estriol y 17-estradiol, que tienen presentaciones comerciales en comprimidos, parches o inyectables. Los estrógenos facilitan la aparición paulatina de ciertos caracteres sexuales secundarios, como el crecimiento de los pechos, la acumulación de grasa en la cadera, la disminución del bello corporal y la obtención de un cutis más fino.

Los transexuales masculinos, hacen una terapia androgénica a base de testosterona que puede suministrarse mediante inyecciones intramusculares, gel 0 parches. Los cambios que pueden obtenerse con la testosterona son aún mayores: el clítoris puede alargarse (permitiendo en algunos casos la penetración), el bello corporal aparece en todo el cuerpo, la voz se hace más grave, se gana masa muscular y la grasa corporal se reestructura, abandonando las caderas para acumularse en la zona abdominal.

El "proyecto corporal" de las personas transexuales es comúnmente referido como eterno y siempre inacabado.

El cuerpo "bay que moldearlo", impedir que se desarrolle, borrar los caracteres secundarios (...) la constancia en la toma de medicación es decisión y responsabilidad del paciente, la terapia bormonal es un primer paso que aliviana y apuntala el trabajo de la cirugía, que viene después (entrevista realizada con Pablo, endocrinólogo) ${ }^{10}$.

El relato de los profesionales nos remite a un paralelo con el cuerpo dócil foucaultiano, que requiere de la propia sujeción corporal a la disciplina y al control, conducido hasta en los más mínimos detalles (DELLACASA, 2013a). De

\footnotetext{
${ }^{10}$ Las notas de campo, indicadas em itálico, así como las fuentes citadas forman parte de la Tesis de Maestría: "Un abordaje antropológico de los procesos diagnósticos, protocolos y rutinas de intervención terapéutica en personas transexuales”, defendida por María Alejandra Dellacasa en la Universidad Buenos Aires en 2013, con la dirección de Alejandra Roca.
} 
este modo, las hormonas se constituyen en un modo de vigilancia eficaz y se tornan en el mejor reaseguro para una identidad sexual "única" y "verdadera", eliminando los caracteres secundarios y con ello cualquier signo de "ambigüedad".

El tratamiento hormonal empieza a funcionar, les va cambiando la vida... el aspecto empieza a mejorar muchísimo, empiezan a hacerse más femeninos o más masculinos, dependiendo el caso, los rasgos empiezan a marcarse, estos rasgos secundarios sexuales se empiezan a marcar para lo que ellos quieren (entrevista realizada con Hebe, endocrinóloga).

La carga hormonal es parte del argumento que justifica y construye la diferencia sexual, hasta el punto que se atribuye "un sexo" a las hormonas sexuales (andrógenos y estrógenos) como si estas fueran exclusivamente masculinas o femeninas y se presentan como determinantes del carácter de la persona. Los tratamientos hormonales que se aplican en los consultorios a personas transexuales se denominan de "reemplazo", reflejando en el plano químico, al igual que en el quirúrgico, un (re)cambio de hormonas "masculinas" por hormonas "femeninas", o viceversa; un proceso químico de "normalización" de la masculinidad y la feminidad (DELLACASA, 2013b).

Sin negar la posibilidad de cambios de humor y del estado emocional producidos, en parte, por el tratamiento hormonal; observamos que en las narraciones de los profesionales, la percepción de ciertos cambios "del carácter" obtenidos con las hormonas está parcialmente condicionada por una visión estereotipada de la masculinidad y la feminidad, que es preexistente al tratamiento. Hay una especie de determinismo hormonal de la personalidad, que emana de las palabras de los médicos y las médicas; una "química del género" en la que lo femenino y lo masculino parece tornarse una cuestión de dosaje sanguíneo. Si bien las hormonas producen una situación fisiológica, siempre subyace una interpretación, consciente 0 inconscientemente, y una serie de creencias acerca de esos resultados.

Es increíble los cambios que se dan en algunas personas, vos la conocés a Érica, yo siempre la pongo de ejemplo, porque cuando vino a verme las primeras veces era muy reo, agresivo, sin modales. y vos la ves abora a ella y es toda delicada, fina... y parece otra persona (entrevista realizada con Hebe, endocrinóloga). 
El poder que se les atribuye a las hormonas deviene de su acción sobre el cuerpo, materializada en actitudes y sobre las que las personas no tendrían ninguna decisión. Muchas veces los efectos de la terapia hormonal se sobrevaloran tanto, que se supone que se conseguirá una identidad de género "acorde" con los genitales (re)creados quirúrgicamente; en algunos casos no sólo se afirma que pueden "crear" mujeres sino además, que éstas pueden cumplir con el ideal de feminidad y belleza de nuestra sociedad.

\section{LAS DISPUTAS POR EL CONOCIMIENTO Y LA INTERVENCIÓN EN LOS CUERPOS}

En su trabajo Testo Yonqui (2008) Beatriz Preciado (en los últimos tiempos conocid@ como Paul B. Preciado) sostiene un auto ensayo corporal y registra las modificaciones somáticas y emocionales que sufre a lo largo de un tratamiento en el que se auto administra testosterona. Testo Yonqui es una especie de diario personal en el que el/la autor/a narra parte de su vida sexual, pero al mismo tiempo, logra plasmar una profunda reflexión acerca de los mecanismos de gestión y dominación somato-política que intervienen en la construcción de nuestro cuerpo y nuestra sexualidad.

Las hormonas no son otra cosa que drogas. Drogas políticas. Como todas las drogas. En este caso, la sustancia no sólo modifica el filtro a través del que descodificamos y recodificamos la realidad, sino que modifica radicalmente el cuerpo y, por lo tanto, el modo en el que somos descodificados por los otros. (PRECIADO, 2008, p. 283)

El acceso, sobre todo a los estrógenos, sin receta médica es relativamente sencillo, puesto que las personas transexuales pueden utilizar los mismos fármacos estrogénicos que utilizan algunas mujeres para la menopausia 0 con fines anticonceptivos. Además, se puede constatar fácilmente la existencia, incluso en palabras de los propios médicos, de circuitos informales de información, compra-venta y administración de hormonas (sobre todo de tratamientos inyectables). Son generalmente las personas transexuales más veteranas (que durante su juventud iniciaron el "proceso transexualizador" sin contar con asesoramiento médico) y aquellas que no quieren someterse a una cirugía genital (y que, por lo general, no acuden al hospital) las que asesoran en el tratamiento a aquellxs que desean comenzar a "hormonarse" y lo hacen, generalmente, sin supervisión médica. Desde la perspectiva de los profesionales, estas prácticas 
desestabilizan el precario equilibrio que se pretende conceder a la idea de la "restitución" de una identidad contrariada por la naturaleza de un cuerpo "equivocado".

(...) explicarles que las hormonas o la medicación que damos para las bormonas no son caramelos, porque si no las vendería el kiosquero. Que yo kiosquera no soy y que necesitamos controles de salud para saber si podemos dar o no determinada medicación, como todas las medicaciones que son fuertes, digamos así... para que ellos lo entiendan (entrevista realizada con Hebe, endocrinóloga).

El uso autoadministrado y recreativo de drogas/hormonas se diferencia del uso "legítimo" de la testosterona o de los estrógenos, como por ejemplo el que está basado en un protocolo de hormonización de una persona diagnosticada como transexual y admitida para un progresivo proceso de "adecuación de sexo". Estos cuerpos, con su autonomía y poca docilidad, constituyen una amenaza a la autoridad científica, eluden controles y singularizan la experiencia con estas tecnologías, de una manera contestataria. En el texto redactado por el Dr. Ricardo Duranti, en la Guía de atención a la salud de personas travestis y transexuales, puede leerse:

El objetivo del tratamiento hormonal en las personas transgénero, travestis en este caso, es suprimir o minimizar los caracteres sexuales secundarios originales e inducir los del sexo buscado (...) A diferencia de lo que sucede con las transexuales, es infrecuente encontrar travestis vírgenes de tratamiento hormonal, lo cual no nos exime de tomar todos los recaudos necesarios para que la continuación del tratamiento se realice en la forma más segura. El deseo y la ansiedad por adaptar el cuerpo a la imagen esperada hace que las personas transgénero tiendan a "hormonarse" cuanto antes maximizando las dosis y siguiendo diversas pautas que, generalmente, son transmitidas de boca en boca, sin control médico. Las personas que solicitan terapia hormonal probablemente gocen de una buena salud y manifiesten un gran apuro por generar cambios corporales, minimizando la necesidad de entrevistas $\mathrm{y} / 0$ estudios previos, lo cual no las exime de un exhaustivo examen general (DURANTI, 2007 p. 2-3).

La web, como espacio público y abierto, ha permitido la vinculación de personas trans de todo el mundo, favoreciendo la circulación de información y el intercambio de experiencias, por fuera de los canales "legitimados" de producción 
de conocimiento. El sitio web "disforia de género"11 es una página gestionada por personas transexuales que reúne artículos e información acerca de la transexualidad y proceso de reasignación de sexo, así como un chat y foros de consulta e intercambio. En una de las secciones, un aviso de tamaño considerable informa:

La auto administración de hormonas es algo potencialmente peligroso que te puede dejar inválida o causar la muerte. Por favor lee este documento a fondo para conocer los riesgos.

A través de diferentes estrategias los cuerpos minoritarios, cuya hibridez ha puesto en cuestión muchas veces su ciudadanía, logran tener acceso a un conjunto de tecnologías que además de intervenir y "moldear" sus cuerpos da lugar a la producción de nuevas subjetividades, nuevas formas de ser y estar en el mundo. En este sentido podemos pensar una redefinición de la distribución y acceso democrático de los recursos dada a partir de la disputa/resistencia a la biomedicina del monopolio de ciertos recursos biotecnológicos, que incluye demandas en torno a la redefiniciones de cuerpo y de identidad sexual.

El proceso de intercambio de propiedades entre humanos y no humanos que tiene lugar en la autoadministración de tratamientos hormonales "cruzados", un sistema sociotécnico conformado por las personas transexuales, los profesionales de la salud, los laboratorios farmacéuticos y las hormonas (en sus diferentes presentaciones comerciales); permite dar cuenta de la co-construcción (JASANOFF, 2004) sociedad-tecnología. Además, da cuenta de procesos en los que la intervención tecnológica se constituye en protagonista, en el devenir de la construcción de la identidad corporal transexual, como herramienta para la fluctuación, construcción y reconstrucción de la subjetividad. La tecnología dispone un sentido utilitario - pragmático y político, que acompaña un tránsito de enunciación subjetiva de condiciones visibles, tales como el ejercicio de una femineidad/masculinidad, la puesta en acto de modelos corporales divergentes 0 incluso la apropiación desafiante y polémica como documenta y exhibe Preciado (2008).

El control cognitivo es indiscernible del control técnico y material; de alguna forma puede decirse que los "objetos" de estudio de los cientistas sociales comenzaron a rebelarse, tomando conciencia de que el "dar nombre" es una

$11<$ http://www.disforiadegenero.org/modules.php?name $=$ News\&file $=$ article\&sid $=340>$ Acceso en septiembre de 2013 
operación política que da lugar a cada uno en un sistema de relaciones, ordenando y jerarquizando los términos y los límites de las discusiones. Los "monstruos" sin voz autorizada - feministas, gays, trans, inmigrantes, pueblos originarios, personas que padecen tal 0 cual síndrome o enfermedad, personas con capacidades diferentes, minorías de todo tipo y calibre - comenzaron a resistir y producir categorías desde sus propios puntos de vista (ROCA, 2012, p. 5). Con el antecedente de los cuestionamientos surgidos desde los movimientos descolonizadores, ambientalistas, estudiantiles y feministas, tal vez uno de los más poderosos signos de estos tiempos, se vincule con la emergencia de sujetos que intentan definir por sí mismos sus problemas y plantean, reclaman o rediseñan soluciones/intervenciones en las formas y modalidades que consideran más adecuadas. Lo que evidencian estos procesos es la matriz política de toda transformación y vivencia corporal, la forma en que los cuerpos capitalizan y despliegan no una mera subjetividad individual sino colectiva, agenciada en la lucha y la capacidad dinámica de producción de identidades situacionales.

En el proceso de constitución de estos "colectivos cognoscentes" (ROCA 2012, p.14) la potestad absoluta e inapelable de la autoridad científica para auspiciar nuevos objetos y asignar nuevos lotes de trabajo académico, comienza a quebrarse, exhibiendo sus maniobras arbitrarias y fugaces.

\section{A MODO DE CIERRE}

Estos últimos años la República Argentina ha sido escenario de debates y cambios radicales en el campo jurídico y sanitario, ya que se ha sancionado una Ley específica que intenta regular la identidad de género ${ }^{12}$ (y aún se encuentra en fase de implementación). Dicha Ley autoriza tanto la rectificación documental de nombre y sexo, como la terapia hormonal de reemplazo y la cirugía de reasignación de sexo, sin necesidad de instancias judiciales, sólo a partir de la propia autodeterminación de las personas. La República Argentina es pionera en una legislación "despatologizante" en esta temática, el Proyecto de Ley que finalmente logró ser sancionado (Proyecto 1879, D-2011), incluye por un lado, el derecho de las personas a decidir respecto de su identidad de género (en lo que hace a nombre y sexo) en los registros oficiales, tanto Documento Nacional de Identidad (DNI) como partida de nacimiento. De este modo habilita a las personas

\footnotetext{
${ }^{12}$ Con este nombre se denomina corrientemente a la Ley 23.746 de Identidad de Género, sancionada en mayo de 2012.
} 
a cambiar la asignación sexo/genérica otorgada por el médico al momento del nacimiento, primando la autopercepción de la persona acerca de su identidad.

Esta experiencia histórica convoca a los sujetos-colectivos que cuestionan y tensionan el punto de vista de los expertos, reclaman una voz propia para denominar, categorizar, establecer o negociar nomenclaturas, intervenir y tomar decisiones; sin la tutela de la ciencia. La "colonialidad" del saber no se disputa sólo desde las categorías filosóficas o sociológicas; los colectivos cognoscentes se organizan sin permiso, resisten, proponen desplazamientos y nuevos sentidos: "varón, mujer, travesti, trans..." la lista de denominaciones que podrían devenir en incorrectas o cuestionadas es infinita e intermitente. Sin embargo, en cada caso impugnan la denominación que los situaba como objetos, se sacuden alguna opresión o escapan de alguna clasificación que resultaba agobiante y que habilitaba intervenciones específicas.

Una serie de dogmas anclados en los dualismos ontológicos modernos: varón-mujer, naturaleza-cultura, normal-patológico, entre otros; resultan cuestionados desde propuestas teóricas que articulan enfoques y perspectivas provenientes de diversos campos empíricos que convergieron en las críticas post positivistas. Entre las más notables se encuentra el planteo de Bruno Latour (1993) quien se ha referido al punto fundacional de la ciencia y la tecnología como la "Constitución Moderna" y describe el trabajo de "purificación" como el esfuerzo por crear dos zonas ontológicas completamente diferenciadas: por un lado el espacio humano, y por el otro lo "no humano"; de un lado un sujeto cognoscente (las palabras en términos de Foucault), y del otro los objetos, las cosas, desprovistas de voluntad, a la espera de "ser conocidos", en una naturaleza estable, objetiva, inmutable y externa.

Entre las más influyentes visiones de esa purificación se encuentra el planteo levistraussiano, que había llevado a suponer la evidencia "real" y universal de lo biológico - naturaleza - disponiendo la autonomía de la cultura como artificio superpuesto y simbólico. Elaine Graham (2002) denomina "higiene ontológica" al establecimiento de fronteras precisas, rígidas y claras entre hombre, naturaleza y máquina. En tanto, la actividad misma de la tecnociencia ha contribuido a exhibir la caducidad de este modelo de conocimiento - basado en la universalidad canónica de los dualismos ontológicos- de modo tal que la crisis de las categorías fundantes del pensamiento moderno (empezando por la noción misma de naturaleza) nos enfrenta al desafío de articular nuevas miradas. Latour (1993, p. 77) identificó esta consecuencia de la tecnociencia como proliferación de híbridos: "mezclas entre géneros de seres enteramente nuevos, híbridos de la 
naturaleza y de la cultura", cuya única forma de relación y organización es la red. Estas redes conformadas por actores, artefactos e instituciones, son dinámicas y flexibles, admiten y permiten registrar la interacción entre sus nodos, tanto objetos como sujetos -humanos y no humanos-, de manera compleja. Latour llama "habitantes de la tierra media" a estos híbridos, precisamente recreando las ideas propuestas por Giovanni Pico della Mirandola y por Giordano Bruno en los siglos XV y XVI:

Te hemos hecho una criatura que no es ni del cielo ni de la tierra, ni mortal ni inmortal, para que puedas, como libre y orgulloso moldeador de tu propio ser, darte a ti mismo la forma que prefieras. Estará en tu poder el descender hacia las más bajas y animales formas de vida; serás capaz, a través de tu propia decisión, de elevarte de nuevo a los órdenes superiores, cuya vida es divina (Discurso sobre la dignidad bumana apud BOSTROM, 2011, p.159).

Los dioses habían dado al hombre inteligencia y manos y lo habían hecho a su imagen, dotándolo con una capacidad superior a la de otros animales (...) esta capacidad consiste no sólo en el poder de trabajar de acuerdo con la naturaleza y el curso normal de las cosas, sino más allá de esto y fuera de sus leyes, con el fin de crear, o tener el poder de crear otras naturalezas, otros cursos, otros órdenes a través de su inteligencia, con la libertad sin la cual el parecido a la deidad no existe (...) los hombres han agudizado su ingenio, han inventado industrias y han descubierto el arte. Y siempre (...) surgen invenciones nuevas y maravillosas. Esto significa que, con su empleo devoto y entusiasta se separan cada vez más de sus naturalezas animales, se elevan cada vez más cerca del divino (G. Bruno, La expulsión de la bestia triunfante apud NOBLE, 1999 p. 56).

Tener el poder de crear otras naturalezas, afirma Giordano Bruno (anterior a Francis Bacon), como una premonición. Autores como Rabinow (1996) señalan que los procedimientos de la ingeniería genética y la biotecnología dan paso a un modelo de naturaleza-manufactura, o biosocialidad, donde la naturaleza deviene artificial, tecnologizada y creada "en" el laboratorio para transformarse en un bien de consumo; además de promover una reescritura 0 corrección de la naturaleza. Las transformaciones corporales y la fragmentación que implican las técnicas mercantilizadas de la biosocialidad establecen nuevos escenarios para pensar los límites de las configuraciones modernas. Según Haraway (1991), son los cyborgs quienes muestran que las fronteras del orden 
moderno se están diluyendo y en su lugar emergen "nuevos límites fluidos e imprecisos, que rompen los dualismos”. Más que un eclipse o un derrumbe del pensamiento moderno (que autorizaría el uso de expresiones como postmodernidad) lo que se revela en este horizonte es una cosmología basada en pactos y convenciones.

En otros trabajos hemos sostenido el carácter eminentemente soteriológico de los relatos centrados en la tecnología (ROCA, 2010, 2006), aquí se trata de una redención de la supervivencia (virtual, sintética 0 tecnológica) del derecho subjetivo a la transformación y la corporificación o encarnación del deseo. El bioensamblaje del cyborg de Haraway circula en el cotidiano, registra cada paso de su transformación y lo comparte públicamente, deja rastros, escenifica y enseña la mutación (como la propia Beatriz devenida en Paul B. Preciado) para dar vida a otros cyborgs, cada vez más "disputantes" de las categorías definidas en espacios cada vez más lábiles y estallados, tales como la ciencia academicista. Una antropología de la ciencia y la tecnología debería entonces, desde su lugar de origen - colonial, periférico, lateral - abocarse a perseguir el fluir de sus monstruos, que ubicados en los "surcos", nos enfrentan a lo inconcebible y tornan evidente la arbitrariedad de nuestras propias clasificaciones.

\section{REFERENCIAS}

BENJAMIN, Harry. The transexual phenomenon. International Journal of Transgenerism, 29 (4), 1966, p. 428-430.

BOSTROM, Nick. A history of transhumanist thought. Journal of Evolution and Technology, 2005, vol. 14, no 1, p. 1-25.

BOSTROM, Nick. Una historia del pensamiento transhumanista. Argumentos de Razón Técnica, $\mathrm{n}^{0}$ 14, 2011, p. 157-191.

BUTLER, Judith. El género en disputa. El feminismo y la subversión de la identidad. Buenos Aires: Ed. Paidós, 2001.

DÍAZ CRUZ, Rodrigo. Al acecho de la perfección. Transhumanismo, el cuerpo oscuro y la vía religiosa de la tecnociencia. Antropología. Boletín Oficial del INAH, (87), 2009, p. 29-45.

DAVIS, Kathy. Reshaping the Female Body: The Dilemma of Cosmetic Surgery. New York: Routledge, 1995. 
DELLACASA, María Alejandra. Un enfoque antropológico de los procesos diagnósticos, protocolos y rutinas de intervención terapéutica en personas transexuales, Tesis de Maestría, Facultad de Filosofía y Letras, Universidad de Buenos Aires, 2013a.

DELLACASA, María Alejandra. Tecnologías biomédicas y producción de cuerpos sexuados: la cirugía de "cambio de sexo" en personas transexuales. Actas de la X Reunión de Antropología del Mercosur - Universidad Nacional de Córdoba, Argentina, 2013b.

D’ODORIC0, María Gabriela. Figuras de lo humano en el nuevo orden tecnológico. Discusiones sobre el devenir político de nuestra especie. Ciencias Sociales: Monstruos y monstruosidades, Revista de la Facultad de Ciencias Sociales de la Universidad de Buenos Aires $N^{0}$ 14, 2014, p. 6-13.

DURANTI, Roberto. Consideraciones específicas sobre el tratamiento hormonal. En: Atención de la Salud de personas travestis y transexuales. Publicación de la Coordinación Sida, GCBA, ONUSIDA, 2007.

ESFANDIARY, Fereidoun. Are you a transhuman?:Monitoring and stimulating your personal rate of growth in a rapidly changing world. New York, NY: Warner Books, 1989.

FIRESTONE, Shulamith. The dialectic of sex; the case for feminist revolution. New York, Morrow, 1971.

FOUCAULT, Michel. La verdad y las formas jurídicas. Barcelona: Gedisa, 1995. FREIDSON, Elliot. La profesión médica. Barcelona: Ed. Península, 1978.

GRAHAM, Elaine. Representations of the Post/Human. Monsters, Aliens and Others in Popular Culture. New Brunswick, New Jersey: Rutgers University Press, 2002.

HARAWAY, Donna. Ciencia, cyborgs y mujeres: La reinvención de la naturaleza. Madrid: Cátedra, 1991.

HUXLEY, Julian. Religion without Revelation. London: Benn, 1927.

JASANOFF, Sheila. States of knowledge: the co-production of science and social order. New York: Routledge, 2004.

LATOUR, Bruno. Nunca hemos sido modernos. Madrid: Debate, 1993.

MANZOCC0, Roberto. Una nuova Torre di Babele. Esseri Umani 2.0. Milan: Springer, 2014. p. 45-116. 
MORE, Max. Transhumanism: Toward a Futurist Philosophy. Extropy 6 (Summer), 1990, p. 6-12.

NOBLE, David. La religión de la tecnología. Buenos Aires: Paidós, 1999.

OUDSHOORN, Nelly. Beyond the Natural Body: An Archeology of Sex Hormones. London: Routledge, London, 1994.

PEARCE, David. The hedonistic Imperative, 2004. Disponible en $<$ www.hedweb.com> Acceso en abril de 2015.

PRECIAD0, Beatriz. Testo Yonqui. Madrid: Espasa Calpe, 2008.

RABINOW, Paul. Science as Practice: Ethos, Logos, Pathos, En: Rabinow, P. Essays of the Anthropology of Reason. New Jersey: Princenton University Press, 1996. REPÚBLICA ARGENTINA, Boletín Oficial. Ley de Identidad de Género. Ley $\mathrm{N}^{\circ}$ 23.743- $\mathrm{N}^{\circ} 32.404,24$ de Mayo de 2012.

ROCA, Alejandra. Del Esencialismo a la Historicidad, enfoques en CET y Género. Dimensiones Sociales de las Nuevas Tecnologías aplicadas a la salud y el cuerpo; su tratamiento en medios gráficos de comunicación masiva. Tesis de Maestría en Política y Gestión de la Ciencia y la Tecnología, Centro de Estudios Avanzados, Universidad de Buenos Aires, 2006.

ROCA, Alejandra. Metáforas y analogías en la construcción de conocimiento: el género y el problema de la neutralidad y autonomía en C\&T. En: Actas de la VII Reunión de Antropología del Mercosur, UFRGS, Porto Alegre, Brasil, 2007.

ROCA, Alejandra. Fragmentos, fronteras y cuerpos incógnitos. Una mirada antropológica sobre la producción y criopreservación de vida en el laboratorio. Tesis de doctorado, Facultad de Filosofía y Letras, Universidad de Buenos Aires, 2010.

ROCA, Alejandra. Todo conocimiento es político: sólo se trata de saber quién es el amo. Revista Espacios No 48, Número especial: Luces y sombras de las Humanidades, Dossier: "Pensar Filo" como "Humanidades", FFYL, UBA, 2012. p. $1-14$.

VILACA Murilo, MARIANO Y PALMA, Alexandre. Límites biológicos, bio-tecnociencia y trans-humanismo: ¿una revolución en Salud Pública? Interface Comunic., Saude, Educ (Botucatu) [online]. Epub, Nov 30, vol.16, n. 43, 2012, p. 1025-1038. 